\title{
Hypercomputation, Unconsciousness and Entertainment Technology
}

\author{
Matthias Rauterberg \\ Industrial Design, Eindhoven University of Technology, The Netherlands
}

\begin{abstract}
Recent developments in computer science introduce and discuss new concepts for computation beyond universal Turing machines. Quantum computing relates to new insights in quantum physics as interference and entanglement based on nonlocality. Several ideas about a new kind of field are presented and discussed. Human unconscious can be interpreted as tapping in these fields for resonating and spreading information. Assuming that culture is based on collective unconscious I propose designing entertainment technology for a new kind of user experience that can influence the individual unconscious and therefore the collective unconscious as well. Our ALICE project can be seen as a first attempt in this direction.
\end{abstract}

Key words: hypercomputation, quantum field, mind, unconscious, culture, entertainment.

\section{Introduction}

This paper discusses an interesting topic: how to use entertainment technology to enhance human life. Several years ago I met Naoko Tosa and got introduced in some of her work, in particular the ZENetic Computer (Tosa et al, 2005; Tosa \& Matsuoka, 2006). Later I was invited by colleagues from Microsoft Research in Cambridge to propose something interesting for possible collaboration. Challenged by this invitation I started to think about a Western equivalent to the Asian ZENetic computer. Luckily the proposed project building an augmented reality system based on the narrative of 'Alice in Wonderland' (Carroll, 1865) got some start-up funding from Microsoft Research. Working hard for more than one year, we -the ALICE team ${ }^{1}-$ achieved a first prototype ready for demonstration (Hu et al, 2008). Still this installation is not on the level of automation as originally planned, now we are at least able to give demos operated by several human 'stage operators'. We could already run a first experiment to test some of our research questions. During the whole project phase and till today a lot of colleagues are asking important questions: Why do you do so? What is the main purpose of this project? What can we gain from it? Although I tried to answer some of these questions already (Rauterberg 1995, 2006a, 2006b, 2006c, 2007), several issues still remain. In this paper I try to provide some post-rationalized and eclectic answers, although being aware of that some of these

${ }^{1}$ For more information see http://www.alice.id.tue.nl/

P. Markopoulos et al. (Eds.): Fun and Games 2008, LNCS 5294, pp. 11-20, 2008.

(C) Springer-Verlag Berlin Heidelberg 2008 
answers might be perceived as speculative, if not inconclusive or even just wrong. Don't worry, lean back, relax and enjoy reading; the 'quantum world' does not like linear causality anyway!

\section{Hypercomputation and the Mind}

The approach to mathematics, to formulate all the axioms needed to deduce all the truths in a particular branch of mathematics mechanistically by removing all unrecognized assumptions based on intuition, became known as the Hilbert Program. This search for axioms became something of a Holy Grail, although Hilbert, who had great respect for the role played by human intuition in the practice of mathematics, never recommended this program named after him (Devlin, 2002). This mechanistically approach to mathematics was complemented with the ideas of Turing (1937, 1938) in which the so called Turing machine was introduced: "A Turing machine is a finite-state machine associated with an external storage or memory medium" (Minsky, 1967, p. 117). The properties of Turing's 'universal machine' have long sustained functionalist intuitions about the nature of cognition. Eliasmith (2002) showed that there is a logical problem with standard functionalist arguments for multiple realizability. He further argued that functionalism is not a useful approach for understanding what it is to have a mind. In particular, it is shown that the difficulties involved in distinguishing implementation from function make multiple realizability claims un-testable and un-informative. As a result, Eliasmith concluded that the role of Turing machines as a concept to conceptualize the mind needs to be reconsidered.

A Turing machine provides a mathematical definition of the natural process of calculating. It rests on trust that a procedure of reason can be reproduced mechanically. Turing's analysis of the concept of mechanical procedure in terms of a finite machine convinced Gödel of the validity of the Church thesis (Church, 1936). Gödel's (1951) later concern was if mental procedures cannot go beyond mechanical procedures as Turing recommended, it would imply the same kind of limitation on human mind. Therefore he decided to treat Turing's argument as inconclusive. The question then is: to which extent a computing machine operating by finite means could provide an adequate model of human intelligence. Lupacchini (2007) argued that a rigorous answer to this question can be given by developing Turing's considerations on the nature of mental processes. For Turing such processes are the consequence of physical processes and he seems to be led to the conclusion that quantum mechanics could help to find a more comprehensive explanation of them. The new view that Turing machines are not the most powerful computing mechanisms was already accepted by Turing himself, who showed in 1939 that his Turing machines with 'oracles' were more powerful than his Turing machines without oracles. This idea of an oracle leads us via non-determinism directly to the uncertainty principle in quantum mechanics of Heisenberg (1930). To go beyond the universal Turing machine, hypercomputation is introduced and discussed (Hanson, 2000; Mulhauser, 
1998, 2001; Ord, 2006) and is also known as super-Turing, non-standard or nonrecursive computation.

Nowadays hypercomputation is the computation of numbers or functions that cannot be computed in the sense of Turing i.e. cannot be computed with paper and pencil in a finite number of steps by a 'human mind' working effectively (Copeland, 2004). Hypercomputers are supposed to solve problems that lie beyond the scope of the standard universal Turing machine by having access to calculation processes with infinite time and to a 'supranatural' memory with infinite information (e.g., Bennett \& DiVincenzo, 2000). Bringsjord and Arkoudas (2004, p167) asked, "one of the things that we don't know is whether the human mind hypercomputes, or merely computes - this despite informal arguments from Gödel, Lucas, Penrose and others - for the view that, in light of the incompleteness theorem, the human mind has powers exceeding those of Turing Machines and their equivalents". So far we know enough to assume that our mind is at least a hypercomputer.

\section{Quantum Physics and Intuition}

Turing used the concept 'machine' for describing mechanical processes and did not emphasize any distinction between a human 'worker-to-rule' and a physical system. If Turing was thinking about mental operations, it is unclear what he thought the brain was doing when it saw the truth in a manner that could not be modeled computably. Turing explained the principle that his universal machine could simulate any machine, if the behavior of these machines is in principle predictable by calculation, in contrast to the indeterminacy principle in quantum mechanics where such kind of predictions is even theoretically impossible. At the end of his life he came up with his 'Turing Paradox': that the standard principles of quantum mechanics imply that in the limit of continuous observation a quantum system cannot evolve. Turing's 'paradox (also know as the 'watched pot problem') in quantum measurement theory, is now called the quantum Zeno effect (Itano, Heinzen, Bollinger \& Wineland, 1990). This effect is relevant to the new technology of quantum 'interaction free measurement' (Hodges, 2008). The quantum Zeno effect states that if we find a system in a particular quantum state, and repeatedly check whether it is still in that state, it will remain in that state. The watched quantum pot never boils (Vaidman, 2008). This looks as if human actions -although indirectly- can have an influence on quantum states (Nelson et al, 2002). Recent experimental evidence in physics suggests quantum non-locality occurring in [sub]conscious and unconscious brain function, and functional quantum processes in molecular biology are becoming more and more apparent. Moreover macroscopic quantum processes are being proposed as intrinsic features in cosmology, evolution and social interactions (Nelson, 2002a, 2002b, 2002c).

Quantum and vacuum physics shows that there is an interconnecting layer in nature, beyond the connectivity we are familiar with. Einstein claimed that no connection between particles can be achieved beyond light speed (the 'local' hypothesis); on the other side it seems to be possible to synchronize quantum states immediately 
(the 'non-local' hypothesis; see Aspect et al, 1982a, 1982b; Aspect, 2007). Bohm (1951) laid most of the theoretical foundations for the Einstein, Podolsky and Rosen (1935) experiments performed by Aspect et al (1982a, 1982b). These experiments demonstrated that if two quantum systems interact and then move apart, their behavior is correlated in a way that cannot be explained in terms of signals traveling between them at or slower than the light speed. This phenomenon is known as nonlocality, and is open to two main interpretations: (1) it involves unmediated, instantaneous action at a distance, or (2) it involves faster-than-light signaling. For if nonlocal connections are propagated not at infinite speeds but at speeds greater than that of light through a 'quantum ether' (a sub-quantum domain where current quantum theory and relativity theory break down) then the correlations predicted by quantum theory would disappear if measurements were made in periods shorter than those required for the transmission of quantum connections between particles. The alternative position is that information always takes time to travel from its source to another location, that information is stored at some para-physical level, and that we can access this information, or exchange information with other minds, if the necessary conditions of 'sympathetic resonance' exist (see also the 'morphic resonance' concept of Sheldrake, 1981).

As for precognition one possible explanation is that it involves direct, 'nonlocal' access to the actual future. Alternatively, it may involve a new kind of perception (beyond the range of ordinary perception) of a probable future scenario that is beginning to take shape on the basis of current tendencies and intentions, in accordance with the traditional idea that coming events cast their shadows before them; such foreshadowing takes place deep in the implicate order which some mystical traditions would call the astral or Akashic realms (Laszlo, 2004). We can assume that there eventually exists an interconnecting cosmic field at the foundation of reality that conserves and conveys information. This cosmic field looks like a possible candidate for our required 'supranatural' memory. Recent discoveries in the field of vacuum physics show that this Akashic field is real and has its equivalent in the zero-point field that underlies space itself. This field consists of a subtle 'sea of fluctuating energies' from which all things arise: atoms and galaxies, stars and planets, living beings, and even [un]consciousness. This zero-point Akashic-field is not only the original source of all things that arise in time and space; it is also the constant and enduring memory of the universe. It holds the record of all that ever happened in life, on Earth, and in the cosmos and relates it to all that is yet to happen.

There are several phenomena like psycho kinesis, telepathy, out-of-body experiences, unidentified flying objects, near death experience, time travel, etc. that are waiting for some explanations (Talbot, 1991). Despite its apparent materiality, the universe looks like a kind of 4-D projection and is ultimately no more real than a hologram, a 4-D image projected in space and time. Using this holographic model Talbot (1991) has developed a new description of reality. It encompasses not only reality as we know it, including hitherto unexplained phenomena of physics, but is capable of explaining such occurrences as telepathy, paranormal and out-of-the- 
body experiences, 'lucid' dreaming and even mystical and religious traditions such as cosmic unity and miraculous healings.

Mitchell (1996) believes that all psychic phenomena involve nonlocal resonance between the brain and the quantum vacuum, and consequent access to holographic, nonlocal information. In his view, this hypothesis could explain not only psycho kinesis and extra sensorial perception, but also out-of-body and near-death experiences, visions and apparitions, and evidence usually cited in favor of a reincarnating soul. One has to admit that these theories are often seen as speculative and not yet part of main stream science.

\section{What is [Un]Consciousness?}

When I think of being conscious, I think of being awake and aware of my surroundings. Being conscious also means being aware of myself as individual. Mostly, people tend to think of being conscious as being alive. If something is breathing and able to move, I think of it as being conscious. If a human being is breathing and able to move, but not able to talk to me, or give me some indication that $\mathrm{s} / \mathrm{he}$ is aware of my presence, I might not be so sure whether the person is conscious. I tend to think that the person should be responsive to the surrounding environment to be conscious. Being in a coma is considered to be the opposite of conscious, so called nonconscious. There are at least three forms of consciousness for humans: (1) the conscious state; (2) the subconscious state; and (3) the unconscious state. In my understanding of 'unconsciousness' I do not really focus on patients which are anesthetized to the best of current ability, with no clinical, EEG, motor or post-operative indications of awareness, or any other similar situations (e.g. coma). The unconscious state I have in mind is full operational and functional for a normal human living as a parallel background process of our mind and body, we are just not aware of (e.g. activities of the cerebellum). The subconscious can be turned into conscious (i.e. by paying attention to subconscious activities); the unconscious normally is not available to the conscious (i.e. the first problem with the unconscious is that it is unconscious). That is, by definition the unconscious represents all that is unknown about us. So how can we talk about something unknown? One solution to the problem is to deny its existence or to not talk about it. To a perfectly logical and rational mind, therefore, the unconscious is just a lot of nonsense! But not for me; let me try to explain why not.

An iceberg can serve as a useful metaphor to understand the unconscious mind (Dijksterhuis, 2007). As an iceberg floats in the water, the huge mass of it remains below the surface. Only a small percentage of the whole iceberg is visible above the surface. In this way, the iceberg is like the mind. The conscious mind is what we notice above the surface while the sub- and unconscious mind, the largest and most powerful part, remains unseen below the surface. The unconscious mind holds all awareness that is not presently in the conscious mind. All activities, memories, feelings and thoughts that are out of conscious awareness are by definition sub- or even unconscious. 
In addition to Freud's individual unconscious (Freud, 1933), Jung has introduced the collective unconscious (Jung, 1934). This is the area of mind where all humanity shares experience and from where we draw on the archetypal energies and symbols that are common to us all (e.g. past life, near death, etc. memories are drawn from this level of the collective unconscious). Another, even deeper level can be described as the universal unconscious where experiences beyond just humanity's can also be accessed with regression process. Jung (1952) coined the term synchronicity, a temporal relationship between causally not related events but experienced as a meaningful coincidence. For Peat (1987) is synchronicity a possible bridge between mind and matter, a way to transform ourselves into getting access to unlimited information. The unconscious connection 'under the iceberg' between people is definitively more powerful than the connection on the conscious level (Dijksterhuis et al., 2006). Although the conscious mind, steeped in cognition and thought, is able to deceive the unconscious mind, based in feeling and intuition, will often give us information from under the iceberg that contradicts what is being communicated consciously (Nakatsu, Rauterberg \& Salem, 2006).

Libet $(1994 ; 1999)$ proposed that the emergent conscious experience be represented in a field, the conscious mental field. The conscious mental field would unify the experience generated by the many neural units. It would also be able to affect certain neural activities and form a basis for conscious will. The conscious mental field would be a new 'supranatural' field. It would be a nonphysical field, in the sense that it could not be directly observed or measured by any external physical means. The conscious mental field theory is outrageously radical, in that it proposes a mode of intracerebral communication which can proceed without requiring neural pathways. The conscious mental field theory provides a 'mechanism' that fits the known properties of conscious experience, including that of conscious free will. It is not dualism, in the Cartesian sense; the conscious mental field does not exist without the living brain, and is an emergent property of that brain.

\section{Discussion and Conclusions}

Looking back, I started discussing the recent outcome to the question 'do humans hypercompute'. This resulted in the concept of hypercomputation beyond universal Turing machines. Hypercomputing seems to be timeless and accessing an infinite memory. A new type of field theory (i.e. Akashic, conscious mental, cosmic, hologram, planetary, quantum, morphic, noosphere, etc.) can provide this supranatural memory. But what has this all to do with entertainment? Nakatsu, Rauterberg and Salem (2006) introduced the concept of Kansei mediation as enabling entertainment technology for cultural transformations (Andrews, 1996). Here is my proposal: let us assume that the human is hypercomputing and this is done unconscious (Dijksterhuis \& Nordgren, 2006; Dijksterhuis et al, 2006). The unconscious has access to a supranatural field and can communicate via resonance in a holistic manner. Let us further assume that the individual unconscious is part of the collective unconscious which is embedded in and forms culture. [see also Burgin, Mark S. "Inductive turing machines." In Doklady Akademii Nauk, vol. 270, no. 6, pp. 1289-1293. Russian Academy of Sciences, 1983.] 
Now I have to introduce my understanding of 'culture' (see also Rauterberg, 2007; Kooijmans \& Rauterberg, 2007). Westerners and East Asians perceive the world and act in it in very different ways (Nisbett et al, 2001; Nisbett \& Masuda, 2003). Westerners pay primarily attention to some focal object, analyzing its attributes and categorizing it in an effort to find out what determinate its behaviour. Determinates used mainly formal logic. Causal attributions tend to focus exclusively on the object and are therefore often mistaken. On the other side, East Asians pay primarily attention to a broad perceptual and conceptual field, noticing relationships and changes and grouping objects based on familiarities rather than categories. They relate causal attributions to the context instead of objects. Mainly social factors are directing the East Asians' attention. They live in complex social networks with determined role relations. Attention to the context is more important than to objects for effective functioning. Westerners live independently in less constraining social worlds and attend to the object and their goals with respect to it. Physical 'affordances' of the environment can also influence perception but is assumed less important. The built environments of the East are more complex and contain more objects than do those of the West. In addition, artistic products of the East emphasize the field and deemphasize objects. In contrast, Western art renders less of the field and emphasizes individual objects and people (Nisbett \& Masuda, 2003).

Cultural theories can be discussed as falling into four focal areas (Keesing, 1974): (1) cultures as adaptive systems, (2) cultures as ideational systems, (3) cultures as socio-cultural systems, and (4) cultures as symbolic systems that are cumulative creations of mind. Conceiving culture as an ideational subsystem within a vastly complex system, biological, economical, social and symbolic, and grounding our abstract models and theories in the creation and usage of artefacts should make it possible to deepen the understanding of ourselves and our future. Whether the concept of culture has to be refined, radically reinterpreted, or progressively extinguished will probably not matter in the long run, unless we can not find a way to ask the right strategic questions, identifying connections that would otherwise be unseen, and therefore to enable us finding the best answers for our cultural development. Therefore ambient culture focuses nowadays on the development of open systems that understand and support the rituals of our living and adapt themselves to people through time and space (Marzano, 2006). Wegner (1997) can show that interactive systems (with a human in the loop) are more powerful than just rule-based machines.

I started the cultural computing project ALICE as an interactive, entertaining experience (see Nakatsu, Rauterberg \& Vorderer, 2005) inspired from 'Alice in Wonderland' (Carroll, 1865). In the scope of this project interactive adventures are experiences provided by an augmented reality environment based on selected parts from Lewis Carroll's book 'Alice's Adventures in Wonderland'. The user assumes the role of Alice and explores this interactive narrative. ALICE is an exploration of interactive story-telling in augmented reality ( $\mathrm{Hu}$ et al, 2008). By exploiting the unique characteristics of augmented reality compared to established media such as film and interactive media, the project uses augmented reality as a new medium for 
edutainment and entertainment as a particular carrier for cultural transformations. Innovations include the refashioning of conventions used in film and interactive tools for the development of an augmented reality narrative, and the use of simple artificial virtual and real characters (avatar and robot respectively) to create an immersive interactive experience.

In ALICE real and virtual agents (i.e. rabbit and caterpillar robot, Cheshire cat) act as characters who lead the user through virtual and real locations, moral choices and emotional states. The narrative is a surreal quest, sometimes funny, sometimes disturbing. The character White Rabbit (representing the concept of time) introduces him and joins with the user in a series of absurdist challenges. ALICE is an educational journey towards the user's heart's desire, designed to provoke self-reflection on a number of other issues: bullying and trusting others; selfish- and selflessness; enjoying the moment or sublimating pleasure. The user is given the opportunity to occupy and experience any of these mental and emotional positions. ALICE can be used to give interesting examples of many of the basic concepts of adolescent psychology. Alice's experiences can be seen as symbolic depictions of important aspects of adolescent development, such as initiation, identity formation, and physical, cognitive, moral, and social development (Lough, 1983). Alice's adventures are de- and reconstructive in nature and as such are directly challenging the strongly held belief of a linear, single track and sequential reality.

Acknowledgements. I am very grateful to my colleagues who made it possible: Sjriek Alers, Dimar Aliakseyeu, Chet Bangaru, Christoph Bartneck, Razvan Cristescu, Jun Hu, Elco Jacobs, Joran Jessurun, Tijn Kooijmans, Hao Liu, Jeroen Peerbolte, Ben Salem, Christoph Seyferth, Vanessa Sawirjo, Joran van Aart, Dirk van de Mortel, Geert van den Boomen, Ton van der Graft, Arrens van Herwijnen, Tijn van Lierop, CheeFai Tan. I have also to thank Microsoft Research in Cambridge and my department of Industrial Design for the financial support.

\section{References}

Andrews, S. (1996). Promoting a sense of connectedness among individuals by scientifically demonstrating the existence of a planetary consciousness? Alternative Therapies, vol. 2, no. 3, pp. 3945.

Aspect A. (2007). Quantum mechanics: to be or not to be local. Nature, vol. 446, pp. 866-867.

Aspect A., Dalibard J. and Roger G. (1982a). Experimental test of Bell's inequalities using timevarying analyzers. Physical Review Letters, vol. 49, no. 25, pp. 1804-1807.

Aspect A., Grangier P. and Roger G. (1982b). Experimental realization of Einstein-Podolsky-RosenBohm Gedankenexperiment: a new violation of Bell's inequalities. Physical Review Letters, vol. 49, no. 2, pp. 91-94.

Bennett CH. and DiVincenzo DP. (2000). Quantum information and computation. Nature, vol. 404, pp. 247-255.

Bohm DJ. (1951). Quantum theory. Prentice-Hall, Englewood Cliffs.

Bringsjord S. and Arkoudas K. (2004). The modal argument for hypercomputing minds. Journal of Theoretical Computer Science, vol. 317, nos. 1-3, pp. 167-190.

Carroll L. (1865). Alice's adventures in wonderland. Macmillan, London. 
Church A. (1936). An unsolvable problem of elementary number theory. American Journal of Mathematics, vol. 58, pp. 345-363.

Copeland BJ. (2004). Hypercomputation: philosophical issues. Theoretical Computer Science, vol. 317 , pp. 251-267.

Devlin K. (2002). Kurt Gödel-separating truth from proof in mathematics. Science, vol. 298, pp. 18991900.

Dijksterhuis A. (2007). Het slimme onbewuste, denken met gevoel. Bert Bakker, Amsterdam.

Dijksterhuis A. and Nordgren L. (2006). A theory of unconscious thought. Perspectives on Psychology, vol. 1, no. 2, pp. 95-109.

Dijksterhuis A., Bos M.W., Nordgren L.F. and van Baaren R.B. (2006). On making the right choice: the deliberation-without-attention effect. Science, vol. 311, pp. 1005-1007.

Einstein A., Podolsky B. and Rosen N. (1935). Can quantum-mechanical description of physical reality be considered complete? Physical Review, vol. 47, no. 10, pp. 777-780.

Eliasmith C. (2002). The myth of the Turing machine: the failings of functionalism and related theses. Journal of Experimental \& Theoretical Artificial Intelligence, vol. 14, no. 1, pp. $1-8$.

Freud S. (1933). New introductory lectures on psychoanalysis, standard edition, vol. 22, Hogarth Press, London.

Gödel K. (1951). Some basic theorems on the foundations of mathematics and their implications (Gibbs Lecture). In: S. Feferman et al (1995, eds.) Collected works III. Unpublished essays and lectures, (pp. 304-323), Oxford University Press, Oxford.

Hanson PP. (2000). Physics, logic and the phenomenal. Minds and Machines, vol. 10, pp. 391-400.

Heisenberg W. (1930). Physikalische Prinzipien der Quantentheorie. Hirzel, Leipzig. [English translation: The Physical Principles of Quantum Theory (1930), University of Chicago Press, Chicago].

Hodges A. (2008). Alan Turing, logical and physical. In: SB. Cooper, B. Löwe and A. Sorbi (eds.) New computational paradigms (pp. 3-15). Springer.

Hu J., Bartneck C., Salem B. and Rauterberg M. (2008). ALICE's adventures in cultural computing. International Journal of Arts and Technology, in press.

Itano WM., Heinzen DJ., Bollinger JJ. and Wineland DJ. (1990). Quantum Zeno effect. Physical Review $A$, vol. 41, no. 5, pp. 2295-2300.

Jung CG. (1934). Die Archetypen und das kollektive Unbewußte. Gesammelte Werke, vol. 9/I, Walter, Olten.

Jung CG. (1952). Synchronicity: an acausal connecting principle. Ark, London.

Keesing RM. (1974). Theories of culture. Annual Review of Anthropology, vol. 3, pp. 73-97.

Kooijmans T., Rauterberg M. (2007). Cultural computing and the self concept: towards unconscious metamorphosis. In: L. Ma, M. Rauterberg, and R. Nakatsu (Eds.): Entertainment Computing ICEC 2007 (LNCS 4740, pp. 171-181), Springer.

Laszlo E. (2004). Science and the Akashic field: an integral theory of everything. Inner Traditions.

Libet B. (1994). A testable field theory of mind-brain interaction. Journal of Consciousness Studies, vol. 1, no. 1, pp. 119-26.

Libet B. (1999). Do we have free will?. Journal of Consciousness Studies, vol. 6, nos. 8-9, pp. 47-57.

Lough GC. (1983). Alice in Wonderland and cognitive development: teaching with examples. Journal of Adolescence, vol. 6, no. 4, pp. 305-315.

Lupacchini R. (2007). Finite machines, mental procedures, and modern physics. Acta Biomedica, vol. 78, no. 1, pp. 39-46.

Marzano, S. (2006.). Ambient culture. In: E. Aarts and J. Encarnação (Eds.) True visions- the emergence of ambient intelligence (pp. 35-52). Springer.

Minsky M. (1967). Computation: finite and infinite machines. Prentice-Hall, Inc., N.J.

Mitchell E. (1996). The way of the explorer: an Apollo astronaut's journey through the material and mystical Worlds. Putnam, New York.

Mulhauser GR. (1998). Mind out of matter: Topics in the physical foundations of consciousness and cognition. Kluwer Academic Publishers, Dordrecht. 
Mulhauser GR. (2001). Reply to Philip P. Hanson's review of 'Mind out of Matter'. Minds and Machines, vol. 11, pp. 301-306

Nakatsu R., Rauterberg M. and Salem B. (2006). Forms and theories of communication: From multimedia to Kansei mediation. Multimedia Systems, vol. 11, no. 3, pp. 304-312.

Nakatsu R., Rauterberg M. and Vorderer P. (2005). A new framework for entertainment computing: from passive to active experience. Lecture Notes in Computer Science, vol. 3711, pp. 1 - 12.

Nelson R. (2002a). EGGs in a global basket. The Golden Thread, no. 5, pp. 8-12.

Nelson R. (2002b). The global consciousness project-part 2. The Golden Thread, no. 8, pp. 6-10.

Nelson R. (2002c). The global consciousness project-part 3. The Golden Thread, no. 11, pp. 30-31.

Nelson RD. Radin DI., Shoup R. and Bancel PA. (2002). Correlations of continuous random data with major world events. Foundations of Physics Letters, vol. 15, no. 6. pp. 537-550.

Nisbett RE. and Masuda T. (2003). Culture and point of view. Proceedings of the National Academy of Sciences, vol. 100, no. 19, pp. 11163-11170.

Nisbett RE., Peng K., Choi I. and Norenzayan A. (2001). Culture and systems of thought: Holistic versus analytic cognition. Psychological Review, vol. 108, no. 2, pp. 291-310.

Ord, T. (2006). The many forms of hypercomputation. Applied Mathematics and Computation, vol. 178, pp. 143-153.

Peat FD. (1987). Synchronicity: the bridge between matter and mind. Bantam, New York.

Rauterberg M. (1995). About a framework for information and information processing of learning systems. In: E. Falkenberg, W. Hesse, A. Olive (eds.), Information System Concepts--Towards a consolidation of views (IFIP Working Group 8.1, pp. 54-69). London: Chapman\&Hall.

Rauterberg M. (2006a). From personal to cultural computing: how to assess a cultural experience. In: G. Kempter \& P. von Hellberg (eds.) uDayIV--Information nutzbar machen (pp. 13-21). Lengerich: Pabst Science Publisher.

Rauterberg M. (2006b). How to assess the user's experience in cultural computing. In: T. Bosenick, M. Hassenzahl, M. Müller-Prove, M. Peissner (Eds.): Usability Professionals 2006 (pp. 12-17). Fraunhofer Informationszentrum Raum und Bau.

Rauterberg M. (2006c). Usability in the future -explicit and implicit effects in cultural computing. In: AM. Heinecke, H. Paul (Eds.): Mensch \& Computer 2006: Mensch und Computer im StrukturWandel (pp. 29-36), Oldenbourg Verlag, München.

Rauterberg M. (2007). Ambient culture: a possible future for entertainment computing. In: A. Lugmayr \& P. Golebiowski (eds.). Interactive TV: a shared experience - Adjunct Proceedings of EuroITV2007. TICSP series \#35 (pp. 37-39), Tampere International Center for Signal Processing, Tampere, Finland.

Sheldrake R. (1981). New science of life: the hypothesis of morphic resonance. Blond \& Briggs, London.

Talbot M. (1991). The holographic universe. HarperCollins, Canada.

Tosa N., Matsuoka S., Ellis B., Ueda H. and Nakatsu R. (2005). Cultural Computing with ContextAware Application: ZENetic Computer. Lecture Notes in Computer Science, vol. 3711, pp. 1323.

Tosa N.and Matsuoka S. (2006). ZENetic Computer: Exploring Japanese Culture. Leonardo, vol. 39, no. 3, pp. 205-211.

Turing AM. (1937). On computable numbers, with an application to the Entscheidungsproblem. Proceedings of the London Mathematical Society, vol. 2-42, pp. 230-265.

Turing AM. (1938). On computable numbers, with an application to the Entscheidungsproblem. A correction. Proceedings of the London Mathematical Society, vol. 2-43, pp. 544-546.

Turing AM. (1939). Systems of logic defined by ordinals. Proceedings of the London. Mathematical. Society, vol. 2, 45, pp. 161-228.

Vaidman L. (2008). Quantum mechanics: evolution stopped in its tracks. Nature, vol. 451, pp. 137-138.

Wegner P. (1997). Why interaction is more powerful than algorithms. Communications of the ACM, vol. 40, no. 5, pp. 80-91. 